\title{
Distributed Fault Detection for Interconnected Second-Order Systems *
}

\author{
Iman Shames, André M. H. Teixeira, Henrik Sandberg, Karl H. Johansson \\ ACCESS Linnaeus Centre, Electrical Engineering, Royal Institute of Technology, Stockholm, Sweden
}

\begin{abstract}
In this paper the existence of unknown input observers for networks of interconnected second-order linear time invariant systems is studied. Two classes of distributed control systems of large practical relevance are considered. It is proved that for these systems one can construct a bank of unknown input observers, and use them to detect and isolate faults in the network. The result presents a distributed implementation. In particular, by exploiting the system structure, this work provides further insight into the design of UIO for networked systems. Moreover, the importance of certain network measurements is shown. Infeasibility results with respect to available measurements and faults are also provided, as well as methods to remove faulty agents from the network. Applications to power networks and robotic formations are presented. It is shown how the developed methodology apply to a power network described by the swing equation with a faulty bus. For a multi-robot system, it is illustrated how a faulty robot can be detected and removed.
\end{abstract}

Key words: Fault Detection and Isolation, Distributed Algorithm, Distributed Detection

\section{Introduction}

Automatic detection of system faults is of growing importance as the size and complexity of systems rapidly increase. Most of the available literature on model-based fault detection and isolation (FDI) focuses on centralized systems where the FDI scheme has access to all the available measurements and the objective is to detect and isolate faults occurring in any part of the system $[5,8,12]$. We further note that while most works dealing with model-based fault detection consider a first-order state space system, it is possible to deal with second-order vector space systems in a fashion akin to that of [7]. Distributed control and monitoring is more suitable than centralized for large-scale interconnected dynamical systems such as power networks and multi-agent systems due to its lower complexity and less use of network resources [18]. Traditional FDI schemes may not be applied to distributed systems, since not all measurements are available in every node.

Some recent work has been done on the design of distributed FDI scheme. In [9], a bank of decentralized observers is built where each observer contains the model of the entire system

\footnotetext{
$\star$ This paper was not presented at any IFAC meeting. Corresponding author I. Shames.

Email addresses: imansh@kth.se (Iman Shames), andretei@kth.se (André M. H. Teixeira), hsan@ee.kth.se (Henrik Sandberg), kallej@kth.se (Karl H. Johansson).
}

and receives both measurements from the local subsystem and information transmitted from other observers. A similar approach is taken in [6] where the observers communicate with each other, but they only possess models of their respective local subsystems. A mixing procedure is used to reconstruct the state of the overall system from the local estimates. Recently a distributed FDI scheme for a network of interconnected first-order systems was proposed. The authors analyzed limitations on fault detectability and isolability in a system theoretic perspective [14].

Power networks are large-scale spatially distributed systems. Being a critical infrastructure, they possess strict safety and reliability constraints [17]. Monitoring the state of the system is essential to guarantee safety. Currently this is typically done in a centralized control center through a single state estimator. The core methodology for state estimation of power systems dates from $1970,[1,16]$. Due to the low sampling frequency of the sensors in these systems a steady state approach is taken, which only allow for an over-constrained operation of the system to ensure reliability. Furthermore faults are handled mainly by hardware devices deployed in the field, so local events leading to cascade failures may pass undetected, since the global state of the system is not taken into account. In recent years, measurement units with higher sampling rate have been developed, e.g. Phasor Measurement Units (PMU), opening the way to dynamic state estimators and observer-based fault detection schemes taking in account the dynamics of the system. Such centralized 
FDI schemes have been proposed in the recent literature, see $[2,15]$. However, to the best of the knowledge of the authors, no distributed method has been proposed to carry out FDI in power networks, despite their inherent decentralized nature.

Contributions. In this paper we address the problem of distributed FDI in a network of nodes with double integrator dynamics, whose interactions are described by a distributed control law. We show how FDI for some power networks and distributed robotic systems fit the problem description. We design continuous-time unknown input observers to achieve the goal. The existence of such observers is established for various conditions on the node interactions under sensing structures. The results are ilustrated on examples in power networks and autonomous mobile node formations.

Outline. The outline of the paper is the following. In Section 2 the problems that are being addressed are formulated. In Section 3 we recall the FDI tools that we use to obtain the main result of this paper. In Section 4 , we propose a solution to the problems posed in Section 2. In Section 5 the application of the results to two practical problems is studied via illustrative examples. Conclusions and suggestions for future work are given in Section 6.

\section{Problem Formulation}

Consider a network of $N$ interconnected nodes and let $\mathcal{G}(\mathcal{V}, \mathcal{E})$ be the underlying graph, where $\mathcal{V} \triangleq\{i\}_{1}^{N}$ is the vertex set, with $i \in \mathcal{V}$ corresponding to node $i$, and $\mathcal{E} \subseteq \mathcal{V} \times \mathcal{V}$ is the edge set of the graph. The undirected edge $\{i, j\}$ is incident on vertices $i$ and $j$ if nodes $i$ and $j$ share a communication link, and a positive weight is associated with this link. Moreover, $N_{i}=\{j \in \mathcal{V}:\{i, j\} \in \mathcal{E}\}$ is the neighborhood set of $i$. Each node $i$ is assumed to have double integrator dynamics

$$
\begin{aligned}
& \dot{\xi}_{i}(t)=\zeta_{i}(t) \\
& \dot{\zeta}_{i}(t)=u_{i}(t)+v_{i}(t),
\end{aligned}
$$

where $v_{i}(t)$ is a scalar known external input, $\xi_{i}, \zeta_{i}$ are the scalar states, and $u_{i}$ is the control given by the linear control law

$$
u_{i}(t)=-\kappa_{i} \zeta_{i}(t)+\sum_{j \in N_{i}} w_{i j}\left[\left(\xi_{j}(t)-\xi_{i}(t)\right)+\gamma\left(\zeta_{j}(t)-\zeta_{i}(t)\right)\right],
$$

where $w_{i j} \in \mathbb{R}_{>0}$, and $\kappa_{i}, \gamma \in \mathbb{R}_{\geq 0}$ for $i, j=1, \ldots, N$. We say that node $k \in \mathcal{V}$ is faulty if for some functions $f_{\xi k}(t)$ and $f_{\zeta k}(t)$ not identical to zero either $\dot{\xi}_{k}(t)=\zeta_{k}(t)+f_{\xi k}(t)$, or $\dot{\zeta}_{k}(t)=u_{k}(t)+v_{k}(t)+f_{\zeta k}(t)$. The functions $f_{\xi k}(t)$ and $f_{\zeta k}(t)$ are denoted fault signals. It is assumed that the faulty node injects fault in only one of the states.

Remark 1. The variables $\xi_{i}$ and $\zeta_{i}$ can be interpreted as position and velocity of node $i$, respectively, for a mobile system, or as phase and frequency in the context of power networks, as further discussed in Section 5.

The closed-loop dynamics of the networked system in the presence of faults can be written as

$$
\begin{aligned}
& \dot{x}(t)=A x(t)+B v(t)+B_{f} f(t) \\
& y(t)=C x(t),
\end{aligned}
$$

where $x(t)=\left[\xi_{1}(t), \ldots, \xi_{N}(t), \zeta_{1}(t), \ldots, \zeta_{N}(t)\right]^{\top} .$. The signal $f(t) \in \mathbb{R}^{m}$ is a vector of unknown fault signals, $y(t) \in \mathbb{R}^{p}$ is the output vector, and $A, B, B_{f}$, and $C$ are matrices of appropriate dimensions. More specifically, we have

$$
A=\left[\begin{array}{cc}
0_{N} & I_{N} \\
-\mathcal{L} & -\gamma \mathcal{L}-\kappa I_{N}
\end{array}\right], B=\left[\begin{array}{c}
0_{N} \\
I_{N}
\end{array}\right]
$$

where $\mathcal{L}$ is the Laplacian matrix and $\kappa=\operatorname{diag}\left(\kappa_{1}, \ldots, \kappa_{\mathrm{N}}\right)$. The $i j$-th entry of $\mathcal{L}, \mathcal{L}_{i j}$, is equal to -1 if $i$ and $j$ share a link and zero otherwise, moreover, $\mathcal{L}_{i i}=-\sum_{j=1, j \neq i} \mathcal{L}_{i j}$. We call the faults $f(t)$ additive faults, see [8].

Before stating the problems, we define what is meant by fault detectability and isolability for systems (3) in the following [8].

Definition 1 (Detectable and Isolable Fault). Given the system (3), $m$ scalar faults $f(t)=\left[f_{1}(t), \ldots, f_{m}(t)\right]^{\top}$ are detectable and isolable if rank $\left[\begin{array}{cc}s I-A & B_{f} \\ C & 0\end{array}\right]=\mathrm{n}+\mathrm{m}$ for almost all $s \in \mathbb{C}$

A fault is thus detectable if the transfer function from $f_{k}(t)$ to $y(t)$ is not identical to zero. Isolable faults relate to input observability and means that any simultaneous occurrence of faults should lead to a change in the output. We further note that the FDI scheme proposed in this paper can detect almost all faults. That is, there may be values of $s \in \mathbb{C}$ for which such matrix has not full rank. Hence there may be some faults generating zero dynamics, which, by definition, do not appear in the system output. These faults cannot be detected using the scheme proposed in this paper.

Note that $B_{f}$ is a matrix such that each of its columns $b_{f_{k}}$ has its entries corresponding to the states of node $k$ as the only non-zero entries. Each node $k$ has a scalar fault signal $f_{k}(t)$ with distribution vector $b_{f_{k}}$. We say node $k$ is faulty if $f_{k}(t)$ is not identical to zero.

The measurement matrix $C$ may be viewed as a design parameter to be chosen in order to ensure the feasibility of the distributed FDI scheme with respect to a predetermined set of faults to be detected. We assume that each node $i$ only measures states within its neighborhood, thus ensuring the distributed nature of the FDI scheme. As it will be shown later on, the specific structure of a feasible local measurement matrix will depend on the faults to be detected. 
In this paper, we solve the following problems:

Problem 1. How can each node of the network detect and isolate a faulty agent?

Problem 2. How can the faulty agent be automatically removed?

We propose a solution to these two problems for two different classes of distributed control laws in the coming sections. In next section we introduce the mathematical tool that we use. Then, in Section 4 we solve Problems 1 and 2, and give conditions for when the solutions exist.

\section{Model-Based Fault Detection Preliminaries}

This paper focuses on observer-based FDI methods. In specific we deal with unknown input observers (UIOs), which have been thoroughly analyzed and developed during the past decade $[5,8]$. We now present UIOs and their application to FDI for centralized linear control systems. A common technique used in model-based fault diagnosis is to generate a set of residuals which indicate the presence of a fault. The residual is a fault indicator computed from the difference between the measurements and their estimates. It should be close to zero if and only if the fault is not present.

Consider the linear fault-free system under the influence of an unknown input $d(t) \in \mathbb{R}^{m-1}$ described by

$$
\begin{aligned}
& \dot{x}(t)=A x(t)+B v(t)+E d(t) \\
& y(t)=C x(t) .
\end{aligned}
$$

The system in presence of faults is given by

$$
\begin{aligned}
& \dot{x}(t)=A x(t)+B v(t)+E d(t)+B_{f} f(t) \\
& y(t)=C x(t) .
\end{aligned}
$$

We assume that the matrices $E$ and $B_{f}$ have full column rank. Remark 2. Note that the condition on $B_{f}$ being full column rank is not restrictive, since any singular matrix $D \in \mathbb{R}^{n \times l}$ can be decomposed in $D=D_{1} D_{2}$, with $D_{1}$ having full column rank. This implies, however, that not all faults are isolable, as follows from the analysis in Section 4.

The matrix $E$ is called a disturbance distribution matrix, since it contains information on how a vector of unknown input disturbances affect the states of the system.

A full-order observer for the fault-free system (5) is described by:

$$
\begin{aligned}
& \dot{z}(t)=F z(t)+T B v(t)+K y(t) \\
& \hat{x}(t)=z(t)+H y(t),
\end{aligned}
$$

where $\hat{x}(t) \in \mathbb{R}^{n}$ is the estimated state and $z(t) \in \mathbb{R}^{n}$ is the observer's state. Note that if we choose $F=A-K C, T=I$, and $H=0$ we have a full-order Luenberger observer. The observer matrices must be designed to achieve the decoupling from the unknown input and meet requirements on the stability of the observer. Choosing the matrices $F, T, K, H$ to satisfy the following conditions

$$
\begin{aligned}
& F=\left(A-H C A-K_{1} C\right), \quad T=(I-H C) \\
& K=K_{1}+K_{2}, \quad K_{2}=F H, \quad(H C-I) E=0,
\end{aligned}
$$

we have the estimation error dynamics

$$
\dot{e}(t)=F e(t) .
$$

where $e(t)=x(t)-\hat{x}(t)$. Now we have the following definition for a UIO.

Definition 2 (UIO). A state observer is a UIO if the state estimation error e $(t)$ approaches zero asymptotically, regardless of the presence of the unknown input $d(t)$.

We conclude that if (8) is satisfied and $F$ is stable, then the observer (7) is a UIO. The following proposition from [5] formalizes this.

Proposition 1. There exists a UIO for (5) if and only if

(1) $\operatorname{rank}(C E)=\operatorname{rank}(E)$

(2) $(C, A-H C A)$ is a detectable pair, where $H$ is given by (8).

For a proof and more details the reader is referred to [5,8]. As suggested in [5], a possible method of detecting and isolating the faults is to use the so called generalized observer scheme (GOS), where we construct a bank of observers generating a structured set of residuals such that each residual is decoupled from one and only one fault, but being sensitive to all other faults. Suppose there is a single fault, $f_{i}(t) \neq 0$. In order to render the observer insensitive to $f_{i}(t)$, this fault is regarded as an unknown input. The system (6) for $d \equiv 0$ is equal to

$$
\begin{aligned}
& \dot{x}(t)=A x(t)+B v(t)+B_{f_{-i}} f_{-i}(t)+b_{f_{i}} f_{i}(t) \\
& y(t)=C x(t),
\end{aligned}
$$

where $b_{f_{i}}$ is the $i$-th column of $B_{f}, f_{i}(t)$ the $i$-th component of $f(t), B_{f_{-i}}$ is $B_{f}$ with the $i$-th column deleted and $f_{-i}(t)$ the fault vector $f(t)$ with its $i$-th component removed. Note that $f_{i}(t)$ can be considered as a disturbance that we want to decouple $\left(b_{f_{i}}\right.$ is analogous to $E$ in (6)). The UIO decoupled from $b_{f_{i}}$ has thus the same structure as (7) and is described by

$$
\begin{aligned}
& \dot{z}_{i}(t)=F_{i} z_{i}(t)+T_{i} B v(t)+K_{i} y(t) . \\
& \hat{x}_{i}(t)=z_{i}(t)+H_{i} y(t) .
\end{aligned}
$$

We introduce residuals to indicated faults.

Definition 3. A residual $r_{i}(t)$ is a fault indicator function that satisfies

$$
\left\|r_{i}(t)\right\|=0 \Leftrightarrow\left\|f_{-i}(t)\right\|=0 .
$$


It is easy to show that we have the following observer error and residual dynamics

$$
\begin{aligned}
\dot{e}_{i}(t) & =F_{i} e_{i}(t)-T_{i} B_{f_{-i}} f_{-i}(t) \\
r_{i}(t) & =C e_{i}(t)
\end{aligned}
$$

where $e_{i}(t)=x(t)-\hat{x}_{i}(t)$ is the observer error and $r_{i}(t)$ is the corresponding residual. Note that the residual dynamics are driven by the $k$-th fault if $T_{i} b_{f_{k}} \neq 0, k \neq i$.

We introduce the following detection and isolation condition for fault $f_{i}(t)$,

$$
\begin{aligned}
\left\|r_{i}(t)\right\| & <\Theta_{f_{i}} \\
\left\|r_{j}(t)\right\| & \geq \Theta_{f_{j}}, \forall j \neq i,
\end{aligned}
$$

where $\Theta_{f_{i}}, \Theta_{f_{j}}>0$ are isolation thresholds, which can be constant or time varying. If (13) is satisfied, we conclude that there is a fault affecting the $i$-th component of the system. Note that the selection of $\Theta_{f_{i}}$ is particularly important. The interested reader may refer to [10] and references there-in for more information

The approach presented above is feasible only if a single additive fault is present. To isolate multiple faults, one can repeat the abovementioned procedure for each of the potential fault combinations. We can derive similar observers for all faults and then use (13) to isolate each of them. Next we show that one can construct UIOs also for classes of networked systems.

\section{FDI for Networked Systems}

In Sections 4.1 and 4.2 we solve Problem 1 of Section 2 by considering two different distributed control laws that are special cases of (2) and show that UIOs can under certain conditions be applied in both cases. Section 4.3 presents the solution to Problem 2.

\subsection{UIO for Position Distributed Control}

Consider the networked system introduced in Section 2 with the following control law

$$
m_{i} u_{i}(t)=-d_{i} \zeta_{i}(t)+\sum_{j \in N_{i}} w_{i j}\left(\xi_{j}(t)-\xi_{i}(t)\right) .
$$

where $m_{i}, w_{i j}, d_{i}>0$. If we make the physical interpretation that $\xi_{i}(t)$ and $\zeta_{i}(t)$ are position and velocity of node $i$, and $m_{i}$ can be interpreted as the agent's mass. The nodes under the control law (14) move towards the position of their neighbors while damping their current velocity.

As in Section 2, assume that

$$
\dot{\xi}_{k}(t)=\zeta_{k}(t)+f_{k}(t)
$$

where $f_{k}(t)$ corresponds to a fault in node $k$. In the presence of this fault, we have

$$
\dot{x}(t)=A x(t)+b_{f}^{k} f_{k}(t)
$$

where $A=\left[\begin{array}{cc}0_{N} & I_{N} \\ -\bar{M} \mathcal{L}-\bar{M} \bar{D}\end{array}\right], \quad B=\left[\begin{array}{ll}0_{N} & \bar{M}\end{array}\right]^{\top}, \bar{M}=$ $\operatorname{diag}\left(\frac{1}{m_{1}}, \cdots, \frac{1}{m_{N}}\right), \bar{D}=\operatorname{diag}\left(d_{1}, \cdots, d_{N}\right)$, and $b_{f}^{k}=$ $\left[\begin{array}{cc}\bar{b}_{f}^{k \top} & 0_{1 \times N}\end{array}\right]^{\top}$ where $\bar{b}_{f}^{k}$ is an $N$ dimensional vector with all zero entries except one that corresponds to the faulty node $k$. Furthermore, we assume the nodes have access to

$$
y_{i}(t)=C_{i} x(t), \quad C_{i}=\left[\bar{C}_{i} 0_{\left|\tilde{N}_{i}\right| \times N}\right], \quad i=1, \ldots, N,
$$

with $\bar{C}_{i}$ being an $\left|\tilde{N}_{i}\right|$ by $N$ matrix with full row rank, where each of the rows have all zero entries except for one entry at the $j$-th position that corresponds to those nodes that are neighbors of $i$, where $\tilde{N}_{i}=N_{i} \cup\{i\}$ and $j \in \tilde{N}_{i}$.

To solve Problem 1, we show that one can construct a UIO at any given node $i$ under the control law (14) using measurements (17).

Theorem 1. Consider the distributed control system with a fault in node $k$ given by (16) and local measurments (17). If $\mathcal{G}$ is connected and $k \in N_{i}$, then there exists a UIO for node $i$.

Proof. First we show that

$$
\operatorname{rank}\left(C_{i} b_{f}^{k}\right)=\operatorname{rank}\left(b_{f}^{k}\right)=1
$$

Denote the row of $C_{i}$ that reads the output of node $k, c_{i}^{k}$. It is obvious that $c_{i}^{k} b_{f}^{k}=1$ and $c_{i}^{j} b_{f}^{k}=0, j \neq k$. Hence, $C_{i} b_{f}^{k}$ is a vector with zero entries except one which is equal to 1 , thus the rank is equal to 1 . This condition is equivalent to condition (1) of Proposition 1.

Then we show that $\operatorname{rank}(\mathcal{D})=2 N+1$ for all $\operatorname{Re}(\mathrm{s}) \geq 0$ where $\mathcal{D}=\left[\begin{array}{cc}s I_{2 N}-A & b_{f}^{k} \\ C_{i} & 0_{\left|\tilde{N}_{i}\right| \times 1}\end{array}\right]$, which is equivalent to to Proposition 1 (2) and also shows the fault is detectable according to Definition 1. We have

$$
\operatorname{rank}(\mathcal{D})=\operatorname{rank}\left[\begin{array}{ccc}
s I_{N} & -I_{N} & \bar{b}_{f}^{k} \\
\bar{M} \mathcal{L} & s I_{N}+\bar{D} \bar{M} & 0_{N \times 1} \\
\bar{C}_{i} & 0_{\left|\tilde{N}_{i}\right| \times N} & 0_{\left|\tilde{N}_{i}\right| \times 1}
\end{array}\right]
$$


Applying some row and column operations we obtain

$$
\operatorname{rank}(\mathcal{D})=\operatorname{rank}\left[\begin{array}{ccc}
0_{N} & -I_{N} & \bar{b}_{f}^{k} \\
a(s) & 0_{N} & b(s) \\
\bar{C}_{i} & 0_{\left|\tilde{N}_{i}\right| \times N} & 0_{\left|\tilde{N}_{i}\right| \times 1}
\end{array}\right],
$$

with $a(s)=s^{2} I_{N}+s \bar{D} \bar{M}+\bar{M} \mathcal{L}$, and $b(s)=\left(s I_{N}+\bar{D} \bar{M}\right) \bar{b}_{f}^{k}$.

We apply a state transformation

$$
\begin{aligned}
& \bar{x}=P x=\left[\xi_{\tilde{i}_{1}}, \cdots, \xi_{\tilde{i}_{\tilde{N}_{i} \mid} \mid}, \xi_{\bar{i}_{1}}, \cdots, \xi_{\bar{i}_{\left|\tilde{N}_{i}\right|}},\right. \\
& \left.\zeta_{\tilde{i}_{1}}, \cdots, \zeta_{\tilde{i}_{\left|\tilde{N}_{i}\right|}}, \zeta_{\bar{i}_{1}}, \cdots, \zeta_{\bar{i}_{\left|\bar{N}_{i}\right|}}\right]^{\top} \text {, }
\end{aligned}
$$

where $\tilde{i}_{j} \in \tilde{N}_{i}, \bar{i}_{j} \in \bar{N}_{i}$, and $\bar{C}_{i}^{*}=\bar{C}_{i} P=\left[I_{\left|\tilde{N}_{i}\right|} 0_{\left|\tilde{N}_{i}\right| \times \bar{N}_{i}}\right]$, where $\tilde{N}_{i}=i \cup N_{i}$ and $\bar{N}_{i}=\mathcal{V} \backslash \tilde{N}_{i}$. After this operation we can write the Laplacian as $\overline{\mathcal{L}}=P^{-1} \mathcal{L} P=\left[\begin{array}{cc}\mathcal{L}_{\left|\tilde{N}_{i}\right|} & l_{\left|\tilde{N}_{i}\right| \times\left|\bar{N}_{i}\right|} \\ l_{\left|\bar{N}_{i}\right| \times\left|\tilde{N}_{i}\right|} & \mathcal{L}_{\left|\bar{N}_{i}\right|}\end{array}\right]$.

Furthermore $P^{-1} \bar{M} P=\left[\begin{array}{cc}\bar{M}_{1\left|\tilde{N}_{i}\right|} & 0_{\left|\tilde{N}_{i}\right| \times\left|\bar{N}_{i}\right|} \\ 0_{\left|\bar{N}_{i}\right| \times\left|\tilde{N}_{i}\right|} & \left.\bar{M}_{2\left|\bar{N}_{i}\right|}\right]\end{array}\right], \quad P^{-1} \bar{D} P=$

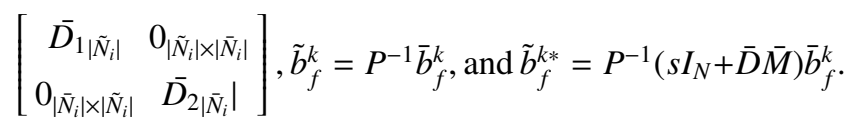

After applying the transformation we have

$$
\operatorname{rank}(\mathcal{D})=\operatorname{rank}\left[\begin{array}{cccc}
0_{|\bar{N}| \times\left|\tilde{N}_{i}\right|} & 0_{\left|\bar{N}_{i}\right| \times\left|\bar{N}_{i}\right|} & -I_{N} & \tilde{b}_{f}^{k} \\
c(s) & \bar{M}_{1} l_{\left|\tilde{N}_{i}\right| \times\left|\bar{N}_{i}\right|} & 0_{\left|\tilde{N}_{i}\right| \times N} & \tilde{b}_{f}^{k *} \\
\bar{M}_{2} l_{\left|\bar{N}_{i}\right| \times\left|\tilde{N}_{i}\right|} & d(s) & 0_{\left|\bar{N}_{i}\right| \times N} & 0_{\left|\bar{N}_{i}\right| \times 1} \\
I_{\left|\tilde{N}_{i}\right|} & 0_{\left|\tilde{N}_{i}\right| \times\left|\bar{N}_{i}\right|} & 0_{\left|\tilde{N}_{i}\right| \times N} & 0_{\left|\tilde{N}_{i}\right| \times 1}
\end{array}\right],
$$

with $c(s)=\bar{M}_{1} \mathcal{L}_{\left|\tilde{N}_{i}\right|}+s^{2} I_{\left|\tilde{N}_{i}\right|}+s \bar{M}_{1} \bar{D}_{1}$, and $d(s)=\bar{M}_{2} \mathcal{L}_{\left|\bar{N}_{i}\right|}+$ $s^{2} I_{\left|\bar{N}_{i}\right|}+s \bar{M}_{2} \bar{D}_{2}$. It is evident that the first and the third columns are independent of the rest, thus

$\operatorname{rank}(\mathcal{D})=\left|\tilde{N}_{i}\right|+N+\operatorname{rank}\left[\begin{array}{cc}\bar{M}_{1} l_{\left|\tilde{N}_{i}\right| \times\left|\bar{N}_{i}\right|} & \tilde{b}_{f}^{k *} \\ \bar{M}_{2} \mathcal{L}_{\left|\bar{N}_{i}\right|}+s^{2} I_{\left|\bar{N}_{i}\right|}+s \bar{M}_{2} \bar{D}_{2} & 0_{\left|\bar{N}_{i}\right| \times 1}\end{array}\right]$

We know from [4] that any principal submatrix of the Laplacian matrix is invertible so the last column is independent of the rest as well, hence $\operatorname{rank}(\mathcal{D})=\left|\tilde{N}_{i}\right|+N+\left|\bar{N}_{i}\right|+1=2 N+1$. This rank equality is equivalent to condition (2) of Proposition 1 [5]. Satisfying the two conditions of Proposition 1 the existence of a UIO for the system (16) with measurements (17) and a fault in node $k$ is established.

Remark 3. Note that if the graph is not connected, the networked system (16) can be decomposed into several decoupled subsystems, each corresponding to a connected subset of the network. The conclusion of Theorem 1 then applies to each subsystem.

The existence of a UIO according to Theorem 1 leads to the possibility to detect a fault at node $k$ from node $i$ using the at methods described in Section 3.

In Theorem 1 we stated that a fault in $\xi_{k}$ can be isolated with the measurements of the form (17). In the next theorem we identify faults that cannot be isolated.

Theorem 2. Consider the system (16). For any of the following pairs of $C_{i}$ and $b_{f}^{k}$, no UIO of the form (7) exists:

(i) $b_{f}^{k}=\left[\begin{array}{ll}\bar{b}_{f}^{k \top} & 0_{1 \times N}\end{array}\right]^{\top}, C_{i}=\left[0_{\left|\tilde{N}_{i}\right| \times N} \bar{C}_{i}\right]$

(ii) $b_{f}^{k}=\left[0_{1 \times N} \bar{b}_{f}^{k \top}\right]^{\top}, C_{i}=\left[0_{\left|\tilde{N}_{i}\right| \times N} \bar{C}_{i}\right]$

(iii) $b_{f}^{k}=\left[\begin{array}{ll}0_{1 \times N} & \bar{b}_{f}^{k \top}\end{array}\right]^{\top}, C_{i}=\left[\bar{C}_{i} 0_{\left|\tilde{N}_{i}\right| \times N}\right]$

Proof. To see that no UIO exists for (i) and (iii), we simply verify that

$$
\operatorname{rank}\left(C_{i} b_{f}^{k}\right)=\operatorname{rank}\left(b_{f}^{k}\right)=0,
$$

so the first condition of Proposition 1 is not satisfied. For (ii), similar to the calculations in proof of Theorem 1 , for the case where $s=0$, we have

$$
\operatorname{rank}(\mathcal{D})=\operatorname{rank}\left[\begin{array}{ccc}
0_{N} & -I_{N} & \bar{b}_{f}^{k} \\
\bar{M} \mathcal{L} & 0_{N} & \bar{D} \bar{M} \bar{b}_{f}^{k} \\
0_{\left|\tilde{N}_{i}\right| \times N} & \bar{C}_{i} & 0_{\left|\tilde{N}_{i}\right| \times 1}
\end{array}\right] .
$$

Recall that $\mathcal{L}$ is rank deficient. Then, it follows that the first column block above is not full column rank. Hence the second condition of Proposition 1 is not satisfied.

Cases (i) and (iii) of Theorem 2 suggest that if there is an unknown input affecting one of the states of one of the nodes in a network, it is not possible to have a UIO without measuring the same state throughout the network as the one affected by the unknown input. For example, if a fault is affecting the velocity of one of the nodes, by measuring positions alone we cannot have a UIO to observe the states of the network. On the other hand, in Case (ii) we see that the first condition of Proposition 1 is satisfied, but a UIO still does not exist. What happens in this case is that the system is not detectable, as seen by observing the first two columns of (18). However, by having access to more measurements one can construct a UIO to detect and isolate faults as seen next.

We now introduce conditions for existence of a UIO to detect the fault

$$
\dot{\zeta}_{k}(t)=u_{i}(t)+v_{i}(t)+f_{k}(t)
$$

where again $f_{k}(t)$ corresponds to a fault in node $k$.

Theorem 3. Consider the distributed control system with a fault in node $k$ given by (16) and local measurments (17) 
with $C_{i}=\left[\begin{array}{cc}\bar{C}_{i} & 0_{\left|\tilde{N}_{i}\right| \times N} \\ 0_{\left|\tilde{N}_{i}\right| \times N} & \bar{C}_{i}\end{array}\right]$, where $\bar{C}_{i}$ is a $\left|\tilde{N}_{i}\right|$ by $N$ matrix, and $b_{f}^{k \top}=\left[\begin{array}{ll}0_{1 \times N} & \bar{b}_{f}^{k \top}\end{array}\right]$ with $b_{f}^{k}$ being an $N$ by 1 vector with $k$-th entry as its only nonzero entry. If $\mathcal{G}$ is connected and $k \in N_{i}$, then there exists a UIO for node $i$.

\subsection{UIO for Position-Velocity Distributed Control}

Now we consider the existence of UIOs for the distributed control law:

$$
u_{i}(t)=\sum_{j \in N_{i}} w_{i j}\left[\left(\xi_{j}(t)-\xi_{i}(t)\right)+\gamma\left(\zeta_{j}(t)-\zeta_{i}(t)\right)\right] .
$$

Again, interpreting $\xi_{i}(t)$ and $\zeta_{i}(t)$ to be position and velocity of node $i$, the nodes under the control law described by (20) move towards the position of their neighbors while penalizing not only the position differences (as previously) but also penalizing the velocity difference. The dynamics of the networked system with a faulty node $k$ is

$$
\dot{x}(t)=A x(t)+b_{f}^{k} f_{k}(t)
$$

where

$$
A=\left[\begin{array}{cc}
0_{N} & I_{N} \\
-\mathcal{L} & -\gamma \mathcal{L}
\end{array}\right],
$$

and $\mathcal{L}$ is the weighted Laplacian matrix with the weight $w_{i j}>0, \gamma>0, b_{f}^{k \top}=\left[\begin{array}{cc}\bar{b}_{f}^{k \top} & 0_{1 \times N}\end{array}\right]$ with $\bar{b}_{f}^{k}$ being an $N$ by 1 vector with $k$-th entry as its only nonzero entry. We further assume that node $i$ measures

$$
y_{i}(t)=C_{i} x(t)
$$

$C_{i}=\left[\begin{array}{cc}\bar{C}_{i} & 0_{\left|\tilde{N}_{i}\right| \times N} \\ 0_{\left|\tilde{N}_{i}\right| \times N} & \bar{C}_{i}\end{array}\right]$, where $\bar{C}_{i}$ is a $\left|\tilde{N}_{i}\right|$ by $N$ matrix of the same structure as considered before. Now we have the following theorem.

Theorem 4. Consider the distributed control system with a fault in node $k$ given by (21) and local measurments (23), and the cases where

$$
\begin{aligned}
& \text { (1) } b_{f}^{k \top}=\left[\begin{array}{ll}
\bar{b}_{f}^{k \top} & 0_{1 \times N}
\end{array}\right] \text {, or } \\
& \text { (2) } b_{f}^{k \top}=\left[\begin{array}{ll}
0_{1 \times N} & \bar{b}_{f}^{k \top}
\end{array}\right]
\end{aligned}
$$

with $\bar{b}_{f}^{k}$ being an $N$ by 1 vector with $k$-th entry as its only nonzero entry. If $G$ is connected and $k \in N_{i}$, then there exists a UIO for node $i$.

Remark 4. Proofs of Theorems 3 and 4 are similar to the proof of Theorem 1 and are therefore omitted.

So far we have established what type of measurements should be available at node $i$ to be able to detect a fault in

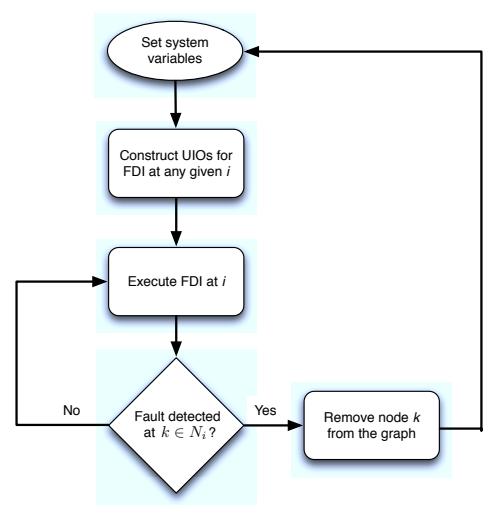

Fig. 1. Faulty Node Removal and Distributed Control Law In the Presence of Fault

$k \in N_{i}$ using a UIO fault detection scheme. More specifically we have shown that if a node aims to detect a fault in a state of one of its neighbors using a UIO based scheme, it has to measure the same state of all of its neighbors. In the next section we address the problem of reconfiguring the distributed control law after detecting a fault in the network.

\subsection{Faulty Node Removal}

In this section, we make the following assumptions for the considered graph. We assume that the graph $\mathcal{G}$ is 2 -vertexconnected, i.e., after losing any single vertex it remains connected. This results in the graph $\mathcal{G}$ to be also 2-edgeconnected, i.e., after losing any single edge it remains connected. Moreover, we consider the case where there is at most one faulty node, $k$, in the formation and the fault is either in $\xi_{k}(t)$ or in $\zeta_{k}(t)$. We propose the algorithm described in Fig. 1 to solve the problem of automatically reconfiguring the distributed control law to cope with a faulty node.

Now, consider the network described in Section 2 with constant external inputs $v$, where $v \neq 0 \in \mathbb{R}^{2 N}$, and the assumptions previously made. Consider the stability of this system where $\dot{x}(t)=A x(t)+v$. A condition on $v$ for the system to converge to an equilibrium point can be identified (entries of $v$ adds to zero.). Note that the algorithm depicted in Fig. 1 cannot be applied to remove the faulty node for such a system with a non-zero input. The reason is that if one applies the algorithm depicted in Fig. 1 after locating the faulty node, $v$ loses one element and the entries of $v$ do not add up to zero anymore, which will drive the system to instability. To remedy this issue, we modify the aforementioned algorithm to deal with removal of the faulty node in such systems, and replace $v_{\ell}\left(\ell \in N_{k}\right)$ by $v_{\ell}+\frac{v_{k}}{\left|N_{k}\right|}$ after removing the faulty node $k$ to ensure convergence to an equilibrium.

\section{Application to Practical Examples}

In this section we consider the problem of fault detection and isolation in two practical problems. First we consider 
detection and isolation of fault in power networks and then we consider the same problem in a formation of mobile nodes with double integrator dynamics.

\subsection{FDI in Power Networks}

In what follows we propose a fault detection and isolation scheme for a power system akin to the one presented earlier. We assume that all the buses in the network are connected to synchronous machines (motors or generators). The behavior of a synchronous electrical motor located in bus $i$ can be described by the so-called swing equation:

$$
m_{i} \ddot{\delta}_{i}(t)+d_{i} \dot{\delta}_{i}(t)-P_{m i}(t)=-\sum_{j \in N_{i}} P_{i j}(t),
$$

where $\delta_{i}$ is the phase angle of bus $i, m_{i}$ and $d_{i}$ are the inertia and damping coefficients, respectively, $P_{m i}$ is the mechanical input power and $P_{i j}$ is the active power flow from bus $i$ to $j$. For more information on the origins of (24) and the reason it is used to describe both load (transmission) buses and generator buses see [11]. Considering that there are no power losses nor ground admittances and letting $V_{i}=\left|V_{i}\right| e^{j \delta_{i}}$ be the complex voltage of bus $i$, the active power flow between bus $i$ and bus $j, P_{i j}$, is given by:

$$
P_{i j}(t)=k_{i j} \sin \left(\delta_{i}(t)-\delta_{j}(t)\right)
$$

where $k_{i j}=\left|V_{i}\right|\left|V_{j}\right| b_{i j}$ and $b_{i j}$ is the susceptance of the power line connecting buses $i$ and $j$.

Since the phase angles are close, we can linearize (25), rewriting the dynamics of bus $i$ as:

$$
m_{i} \ddot{\delta}_{i}(t)+d_{i} \dot{\delta}_{i}(t)=-\sum_{j \in N_{i}} k_{i j}\left(\delta_{i}(t)-\delta_{j}(t)\right)+P_{m i}
$$

Consider a power network with $\mathcal{G}(\mathcal{V}, \mathcal{E})$ as its underlying graph with $N=|\mathcal{V}|$ nodes, where each node corresponds to a bus in the power network. Rewriting (26) in state-state form and considering $x=\left[\delta_{1}(t), \cdots, \delta_{N}(t), \dot{\delta}_{1}(t), \cdots, \dot{\delta}_{N}(t)\right]^{\top}$ and $v(t)=\left[\begin{array}{lll}P_{m 1} & \cdots & P_{m N}\end{array}\right]^{\top}$, we have

$$
\dot{x}(t)=A x(t)+B v(t)
$$

where $A=\left[\begin{array}{cc}0_{N} & I_{N} \\ -\bar{M} \mathcal{L} & -\bar{M} \bar{D}\end{array}\right], B=\left[\begin{array}{ll}0_{N} & \bar{M}\end{array}\right]^{\top}, \bar{M}=$ $\operatorname{diag}\left(\frac{1}{m_{1}}, \cdots, \frac{1}{m_{N}}\right), \bar{D}=\operatorname{diag}\left(d_{1}, \cdots, d_{N}\right)$.

Consider that the network is being affected by faults corresponding to unexpected changes in the power generation or consumption. Assume that a fault has occurred at node $k$.

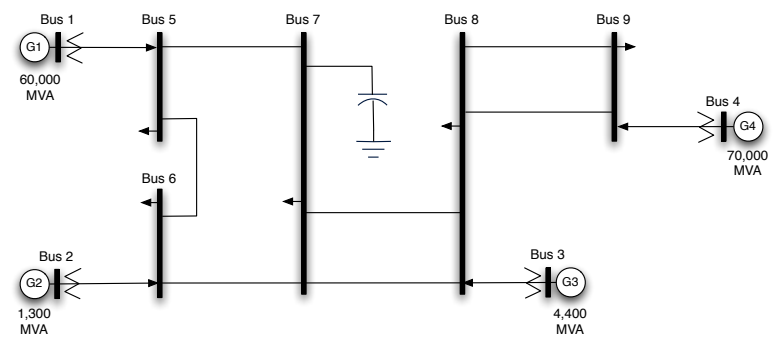

Fig. 2. Power network with 9 buses [3].

The power network under such conditions can be modeled as

$$
\dot{x}(t)=A x(t)+B v(t)+b_{f}^{k} f_{k},
$$

where $b_{f}^{k}$ is the $k$-th column of $B$ and therefore it can be written as $b_{f}^{k}=\left[\begin{array}{ll}0_{1 \times N} & \bar{b}_{f}^{k \top}\end{array}\right]^{\top}$ with $\bar{b}_{f}^{k \top}$ being a column vector with $\frac{1}{m_{k}}$ in the $k$-th entry and zero in all other entries. Thus, from Theorem 3 there exists a UIO for such system at a given node $i$ if $k \in N_{i}$ and $y_{i}=C_{i} x$ with

$$
C_{i}=\left[\begin{array}{cc}
\bar{C}_{i} & 0_{\left|\tilde{N}_{i}\right| \times N} \\
0_{\left|\tilde{N}_{i}\right| \times N} & \bar{C}_{i}
\end{array}\right]
$$

Thus we need to measure the phase and frequency of the neighbors to be able to detect the faulty node. These measurements are readily available through phase measurement units (PMU). Having such measurements, this type of faults can be detected and isolated in a distributed way using UIOs. Remark 5. Because of Theorem 2 we know that we cannot solve the fault detection problem using UIO with having access to less information than the information available through $y_{i}=C_{i} x$, with the above-mentioned $C_{i}$.

Remark 6. In the case where there are buses that are not connected to synchronous machines and are described by algebraic equations; one has two alternatives. First, one can use equation (24) to model only the buses that are connected to synchronous machines and use the techniques in [13], Chapter 14, to remove the algebraic relations from the power network model and assume that the faults only affect the buses connected to synchronous machines. Second, one may assume that the buses that are not connected to the machines are governed by dynamic equations of type (24), albeit with small damping and inertia coefficients [11].

Consider the power network presented in Fig. 2. The power grid's topological parameters and the generators' dynamic coefficients $\left(m_{i}\right.$ and $\left.d_{i}\right)$ were taken from [3], while the dynamic coefficients of the rest of the buses were arbitrarily taken from reasonable values.

The power network is evolving towards the steady-state when, at time instant $t=2 s$, a fault occurs at node 6 , as presented in Fig. 3(a). By implementing a bank of observers 


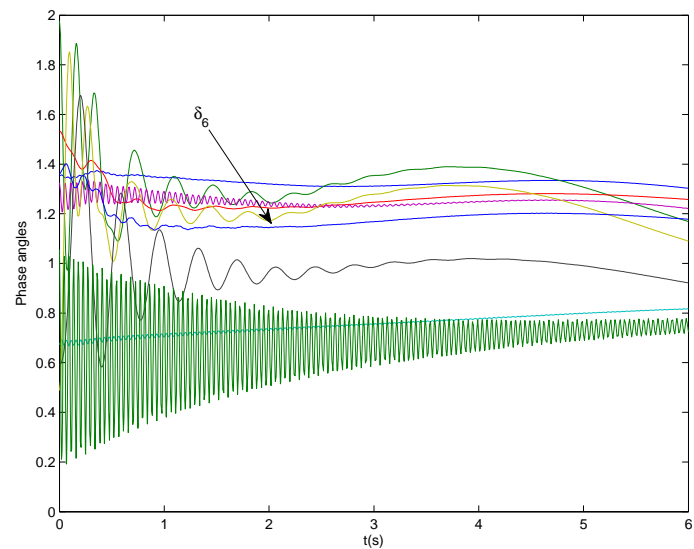

(a)

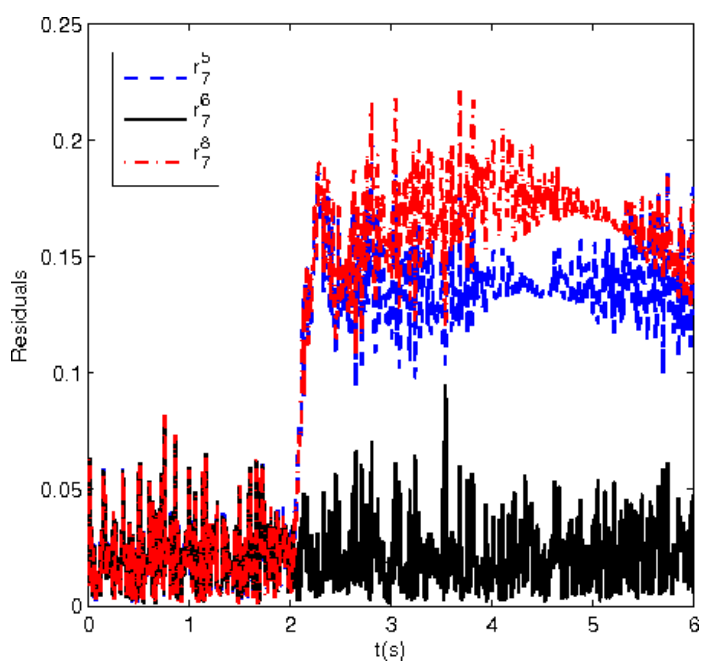

(b)

Fig. 3. FDI in a Power Network: (a) Phase angles of the power network. (b) Residuals of buses neighboring bus 7 .

at bus 7 , the fault is successfully detected and isolated in the presence of process and measurement noise, since the residual corresponding to bus 6 became larger than the other residuals, as illustrated in Fig. 3(b).

\subsection{FDI in Formations of Mobile Agents}

In this section adopt the system and the notations introduced in Section 4.2. Furthermore, assume at time $t_{f}$ a fault occurs at node $k$, one can detect and isolate this fault using the methods introduced earlier.Consider a formation consisting of 10 nodes with double integrator dynamics with the aforementioned control law as depicted in Fig. 4(a). Further assume at time $t_{f}=2$ node 3 starts to malfunction. Using UIOs and the logic presented in (13) this fault is detected at time $t_{d}=3.56$. A sample of residuals as calculated in node 1 (neighbouring node 3) is presented in Fig. 4(b). In the case where no isolation is carried out the first coordinate of the velocities of the nodes are presented in Fig. 4(c). However, if after the detection of the fault, the aforementioned algo- rithm is used to remove the faulty node, the first coordinate velocities of the nodes would be as the ones depicted in Fig. 4(d). Which shows that they have reached consensus. Due to absence of any external input it is not needed to adjust external input after disconnection.

\subsection{Complexity of the FDI Method}

For implementation of the method introduced in this paper, at each node it is required to have one observer corresponding to each of the neighbours. Each of these observers have $2 N$ states. So at each node $i, 2 N\left|N_{i}\right|$ states are estimated, which puts a heavy computational burden on each of the nodes as $N$ increases. In particular, the example in Section 5.1 with 9 nodes required node 7 to have 3 observers with 18 states each, corresponding to 54 states for the observer bank. As for the example in Section 5.2 with 10 nodes the observer bank in node 1 , having 5 neighbors, would require a total of 100 states.

However, we note that it is not required to (i) estimate all the states in the system at each observer, and (ii) it is not necessary to have observers at all of the nodes. These cases are discussed in [19].

\section{Concluding Remarks and Future Directions}

In this paper we considered the problem of fault detection and isolation in the networks of interconnected nodes with double integrator dynamics. We proposed a distributed FDI scheme based on UIOs requiring only local measurements. Furthermore we analyzed the feasibility of such scheme with respect to local measurements and we also provided some infeasibility results. As part of a mitigation procedure, we proposed an algorithm to remove the faulty node from the network that can also be applied when there are nonzero external inputs. Then we presented some simulation examples related to the motivating applications, thus demonstrating the application of the proposed method to fault detection in power and multinode systems. Some considerations on the complexity and scalability of the proposed method were also given.

Possible future directions include considering a way to reduce the dimension of the unknown input observers at each node in the current scheme, and explore applicability of other fault detection methods to the problems considered here that are more robust to the noise.

\section{Acknowledgements}

This work was supported in part by the European Commission through the VIKING project, the Swedish Research Council, the Swedish Foundation for Strategic Research, the Knut and Alice Wallenberg Foundation, and NICTA, which is funded by the Australian Government as represented by the Department of Broadband, Communications 


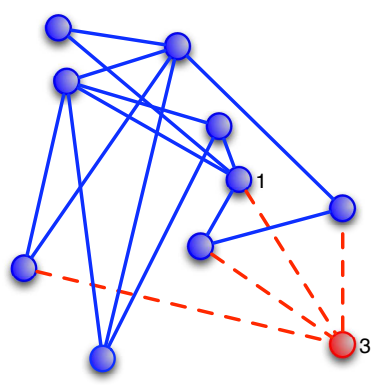

(a) The formation of 10 nodes.

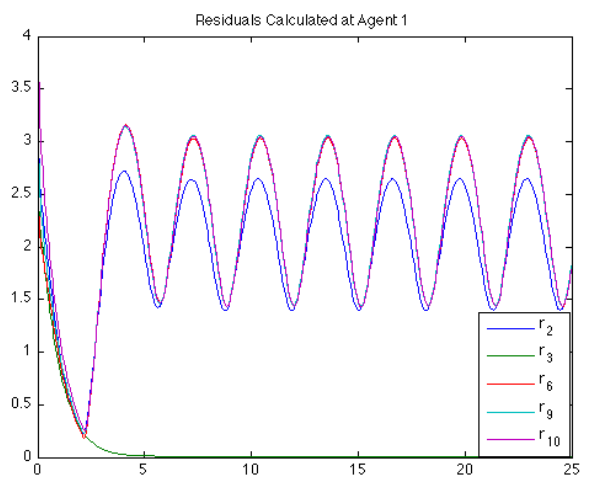

(b) Residuals of nodes neighbouring node 1 .

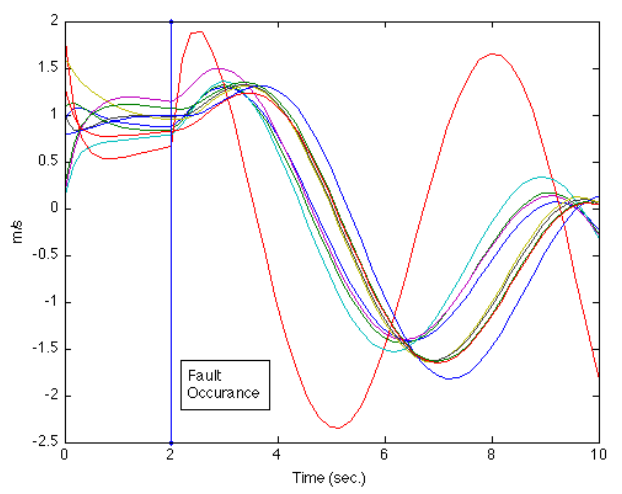

(c) First coordinate of the velocities of the nodes when the faulty node is not removed.

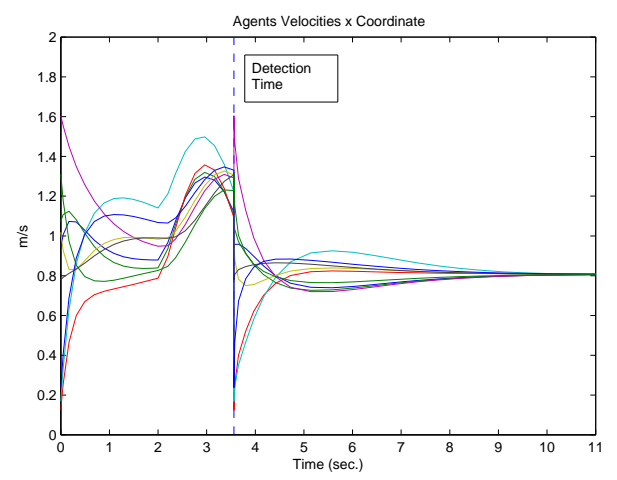

(d) First coordinate of the velocities of the nodes when the faulty node is removed.

Fig. 4. Multi-agent formation in the presence of a fault occurring at $t=2 \mathrm{sec}$. in node 3 . and the Digital Economy and the Australian Research Council through the ICT Centre of Excellence program.

\section{References}

[1] A. Abur and A.G. Exposito. Power System State Estimation: Theory and Implementation. Marcel-Dekker, 2004.

[2] M. Aldeen and F. Crusca. Observer-based fault detection and identification scheme for power systems. In IEE Proceedings Generation, Transmission and Distribution, volume 153, pages 7179, January 2006.

[3] P. M. Anderson and R. G. Farmer. Series compensation of power systems. PBLSH. Inc, California, USA, 1996.

[4] P. Barooah and J.P. Hespanha. Graph effective resistance and distributed control: Spectral properties and applications. In Decision and Control, 2006 45th IEEE Conference on, pages 3479-3485. IEEE, 2007.

[5] J. Chen and R. J. Patton. Robust Model-Based Fault Diagnosis for Dynamic Systems. Kluwer Academic Publishers, 1999.

[6] W. H. Chung, J. L. Speyer, and R. H. Chen. A decentralized fault detection filter. Journal of Dynamic Systems, Measurement, and Control, 123(2):237-247, 2001.

[7] M. A. Demetriou. Using unknown input observers for robust adaptive fault detection in vector second-order systems. Mechanical systems and signal processing, 19(2):291-309, 2005.

[8] S. X. Ding. Model-based Fault Diagnosis Techniques: Design Schemes. Springer Verlag, 2008.

[9] S. X. Ding, P. Zhang, Ch. Chihaia, W. Li, Y. Wang, and E. L. Ding. Advanced design scheme for fault tolerant distributed networked control systems. In Proceedings of the 17th IFAC World Congress, pages 13569 - 13574, Seoul, Korea, July 2008.

[10] P. M. Frank and X. Ding. Survey of robust residual generation and evaluation methods in observer-based fault detection systems. Journal of process control, 7(6):403-424, 1997.

[11] R. B. L. Guedes, F. Silva, L. F. C. Alberto, and N. G. Bretas. Large disturbance voltage stability assessment using extended Lyapunov function and considering voltage dependent active loads. In Power Engineering Society General Meeting, 2005. IEEE, pages 1760-1767. IEEE, 2005.

[12] R. Isermann. Model-based fault detection and diagnosis: status and applications. In Proceedings of the 16th IFAC Symposium on Automatic Control in Aerospace, pages 71-85, St. Petersburg, Russia, June 2004.

[13] J. Machowski, J.W. Bialek, and J.R. Bumby. Power system dynamics: stability and control, volume 12. Wiley, 2008.

[14] F. Pasqualetti, A. Bicchi, and F. Bullo. Consensus computation in unreliable networks: A system theoretic approach. IEEE Transactions on Automatic Control, 2010. Submitted, available online at http://www.fabiopas.it/papers/FP-AB-FB-10a.pdf.

[15] E. Scholtz and B.C. Lesieutre. Graphical observer design suitable for large-scale DAE power systems. In Proceedings of the IEEE Conf. on Decision and Control, pages 2955-2960, Cancun, December 2008.

[16] F. C. Schweppe and J. Wildes. Power system static-state estimation, part I: Exact model. IEEE Transactions on Power Apparatus and Systems, 89(1):120-125, January 1970.

[17] M. Shahidehpour, W. F. Tinney, and Y. Fu. Impact of security on power systems operation. Proceedings of the IEEE, 93(11):20132025, November 2005.

[18] D. D. Siljak. Decentralized control of complex systems. Academic Press, 1991.

[19] A. Teixeira, H. Sandberg, and K. H. Johansson. Networked control systems under cyber attacks with applications to power networks. In Proc. American Control Conference, 2010. 